(Aus der Königl. Universitäts-Frauenklinik zu Leipzig.)

\title{
Ueber einen Fall von Neuroma verum gangliosum amyelinicum des Bauchsympathicus.
}

\author{
Von \\ Dr. Adolf Glockner, \\ Assistenten der Königl. Universitäts-Frauenklinik zn Leipzig. \\ (Mit 4 Abbildungen auf Tafel VII.)
}

Vor Kurzem wurde in der hiesigen Klinik von Herrn Geheimrath $Z_{w}$ eifel ein retroperitonealer Tumor exstirpirt, welcher sich bei der genauen mikroskopischen Untersuchung als echtes gangliöses Neurom des Sympathicus erwies.

Die Geschwulstspecies der Neurome ist bekanntlich von Virchow in den "krankhaften Geschwülsten" aufgestellt und sind dort die verschiedenen Arten derselben näher charakterisirt worden.

Virchow hat zugleich auch an dieser Stelle eine derartige Geschw̧ulst genau beschrieben; Ganglienzellen enthielt dieselbe allerdings nicht und nimmt Virchow zum Vorkommen derselben in Neuromen eine ablehnende Stellung ein, da ihm die bis damals bekannten einschlägigen Fälle einer strengen Kritik nicht Stand zu halten schienen. Seither wurden eine Reihe von gangliösen Neuromen veröffentlicht, unter der Zahl welcher die Fälle von Loretz ${ }^{1}$, Weichselbaum ${ }^{2}$ ), Axel Key ${ }^{3}$, Borst ${ }^{4}$ ), Beneke ${ }^{5}$ ),

1) Virchow's Arch. Bd. 49.

2) Ebendas. Bd. 85 .

3) Ref. Virch.-Hirsch. 1880.

4) Würzburger Sitzungsber. 28. 10. 1897.

5) Centralbl. f. pathol. Anatom. 1897. S. 846 . (Naturforscherversammlung, 2 Fälle.) 
Knauss $^{1}$ ), Chiari' $^{2}$ ), Busse ${ }^{3}$ ), M. B. Schmidt ${ }^{4}$ ) als sichergestellt gelten können.

Bei der geringen Zahl der bekannten Fälle von Neuroma verum gangliosum mag die kurze Mittheilung des nachfolgenden Falles berechtigt erscheinen, zumal da derselbe auch klinisch interessante Eigenthümlichkeiten zeigt.

Die Anamnese ergab folgendes:

H. W., 38 Jahre alt, Strumpfwirkersfrau, Mutter an Magenkrebs gestorben. Die ersten Menses traten mit 16 Jahren auf, waren immer unregelmässig, bis zu einem halben Jahre aussetzend; letzte Menses vor 14 Tagen.

8 normale Entbindungen, Wochenbetten immer gut verlaufen. Von den Kinder sind 2 klein gestorben, 6 leben, sind jedoch kränklich. Pat. leidet häufig an anfallsweise auftretenden heftigen Schmerzen in der linken Nierengend, ausserdem an Kopfschmerz und Schwindel. Seit einigen Monaten besteht weisslicher Ausfluss in mässiger Stärke. Der Stuhlgang ist unregelmässig, Appetit gering; Erbrechen besteht nicht; Pat. will in den letzten Monaten ziemlich stark abgemagert sein.

Status praesens: Gracil gebaute Frau mit gering entwickelter Muskulatur und Panniculus adiposus, Herz- und Lungenbefund normal. Leber nicht vergrössert, rechte Niere deutlich palpabel. Im Hypogastrium Druckempfindlichkeit. Linke Niere nicht mit Sicherheit zu fühlen. In der linken Seite, etwas oberhalb der Linea innominata, ist eine schwach faustgrossse, ziemlich derbe, annähernd eiförmige Geschwulst fühlbar, welche sich nach oben sowie seitlich leicht verschieben lässt und auf Druck etwas empfindlich ist.

Der Genitalbefund war, abgesehen von eine Erosion der Portio vaginalis, ein durchaus normaler, insbesondere liess sich bestimmt nachweisen, dass der erwähnte Tumor in keinerlei Zusammenhang mit den Genitalien steht. Der Urin war frei von Eiweiss und Zucker.

Bei Aufblähung des Dickdarmes mit Luft oder bei Füllen desselben mit Wasser verschwindet der Tumor hinter dem geblähten Dickdarme und ist palpatorisch nur noch unsicher nachweisbar.

Irgend welche Störungen der Sensibilität, Motilität oder des Verhaltens der Reflexe bestehen nicht.

Eine sichere Diagnose war bei diesem wenig charakteristischen Befunde nicht zu stellen; in erster Linie wurde an einen Tumor des Darmes oder retro-peritonealen Tumor gedacht. Da nach den anamnestischen Angaben der Pat. mit einer möglicherweise malignen Geschwulst gerechnet werden musste, wurde Probe-Laparotomie beschlossen.

Operation am 7. 11. 1900 (Geheimrath Zweifel):

Nach Eröffnung der Bauchhöhle zeigt sich ein apfelsinengrosser, sehr beweglicher Tumor, welcher, von dem Mesenterium einer Dünndarmschlinge umschlossen, an der Wirbelsäule, etwas links von der Mittellinie, zu inseriren scheint. Leider wurde während der Operation

1) Virch. Arch. Bd. 153.

2) Centralbl. f. pathol. Anatom. 1897. S. 847.

3) Virch. Arch. Bd. 151. (Supplement.)

4) Ebendas. Bd. 155. - Ueber die sonstige Literatur cfr. Knauss l. c. 
der Insertionspunkt der Geschwulst an der Wirbelsäule nicht genauer festgestellt; derselbe dürfte etwa im Niveau des Körpers des 3. Lendenwirbels (entsprechend dem Ansatze der Radix mesenterii) gelegen gewesen sein. Nach Spaltung des Mesenteriums lasst sich der Tumor ziemlich leicht stumpf aus seinem Bette ausschälen, mehrfach treten von den beiden Mesenterialblättern kleinere Gefässe an den Tumor heran, welche durchtrennt und unterbunden wurden. Beim weiteren Auslösen der Geschwulst lässt sich eine Art Stiel bilden, welcher sich direct auf der Seitenwand der Aorta anzusetzen scheint. In diesem Stiele fühlt man die Pulsation einer starken Arterie. Der Stiel wird mit einer Collin'schen Klemme gefasst, der Tumor darüber abgetragen. Hierauf wird der Stiel durch Partienligaturen versorgt, nachdem zuvor noch die erwähnte 'Arterie, welche ungefähr das Kaliber einer Radialis hat, isolirt unterbunden war. Es zeigt sich nun noch ein kleiner Rest von Geschwulst auf dem Stiele aufsitzend, welcher sich indess leicht abtragen lässt. - Der abgebundene Stiel selbst besteht jetzt nur noch aus einem starken Gefässbündel und einer dasselbe einhüllenden Bindegewebslage. Nervenstämme warèn in diesem Bändel makroskopisch nicht zu erlkennen.

Die den Stumpf versorgenden Partienligaturen sind etwa $2 \mathrm{~cm}$ von der Aorta entfernt. Die dem Geschwulstbett entsprechende Tasche im Mesenterium wurde durch eine fortlaufende Catgutnaht zusammengerafft, die Mesenterialincision vernäht und nach Trockenlegung der Bauchhöhle dieselbe in der an der hiesigen Klinik üblichen Weise geschlossen.

Nach dem Erwachen aus der Narkose klagt Pat. ïber starke Schmerzen, es besteht mässig starkes Aufstossen und Erbrechen.

In der Nacht wird das Aufstossen stärker;" gegen 3 Uhr früh collabirt Pat. plötzlich, der Puls wird sehr klein, ist zeitweise kaum fühlbar. Erbrechen grünlicher Massen. Subcutan wird Kampheröl gegeben, auf das Abdomen werden 2 Sandsäcke gelegt.

Das Erbrechen dauert an, Puls an der Radialis kaum fühlbar, an der Carotis gezählt 126 Schläge, Temperatur $36,6-36,9^{\circ}$. Pat. sieht sehr verfallen aus.

Auf 'Infusion von 11 physiologischef Kochsalzlösung wird der Puls vorübergehend besser, ebenso nach den halbstündlich erfolgenden Kampherinjectionen.

Gegen Mittag: wird der Zustand wiederum schlechter; der Leib ist wenig aufgetrieben, mässig druckempfindlich, Zunge trocken, borkig, Puls an der Carotis 136 , Temperatur 36,90. Da an eine Undurchgängigkeit des Darmrohres gedacht wurde, wurde zur Prüfung derselben ein hohèr Einlauif gemacht.

Ein nachgiebiges Gummirohr liess sich ohne Schwierigkeit etwa $30 \mathrm{~cm}$ weit einführen. Das eingegossene Wasser kommt kaum gefärbt zurück. Etwa eine Viertelstunde später, Abgang von reichlichen Flatus mit etwas Stuhl. Pat. fühlt sich sehr erleichtert, schläft nachmittags etwas. Puls 136, ist etwas kräftiger geworden: es besteht noch ziemlieh viel Aufstossen, Schmerzen sind mässig.

Am 9. 11. mässig viel Aufstossen, kein Erbrechen.

Temp. 36,7-37,70, Puls leidlich $110-120$. Pat. kann kein Wasser lassen, muss katherisirt werden.

10. 11. Wenig Aufstossen, Puls ziemlich kräftig, 88-92, Temperatur $36,8-37,4^{\circ}$. Nach Einlauf reichlich Stuhl.

11. 11. Fast kein Aufstossen mehr. Puls 80-90, Temp. 37,0 bis $37,5^{\circ}$. Der Verlauf ist von jetzt ab ein völlig ungestörter. 
Pat. steht am 17. Tage auf und wird 2 Tage darauf, am 26.' 11. geheilt entlassen.

Am 26. 1. 1901 stellte" sich Pat. zur Nachuntersuchung vor.

Die Bauchnarbe ist fest, der Genitalbefund normal. Bezüglich ihrer Beschwerden giebt Pat. an, dass die Magenschmerzen zwar zeitweise noch augetreten seien, aber in viel schwächerem Maasse als früher.

Der exstirpirte Tumor ist ungefähr eiförmig, misst in der Länge $8 \mathrm{~cm}$, in der Breite $6,5 \mathrm{~cm}$ und in der Dicke $5 \mathrm{~cm}$. Die erwähnte grosse Arterie tritt an der hinteren Fläche, nahe, dem unteren. Pol in die Geschwulst ein. Der Tumor zeigt eine vollkommen glatte Oberfläche, welche allenthalben von einer dünnen Lage von Binde- resp. Fettgewebe bedeckt ist. Nirgends sind an der Oberfläche mikroskopisch erkennbare Nervenstämme wahrzunehmen. Auf dem Durchschnitte sieht man als periphere Begrenzung eine deutliche, aber nur sehr dünne fibröse Kapsel, welche sich von dem unterliegenden Gewebe, theilweise wenigstens, stumpf Ioslösen lässt. Die ziemlich derbe, röthlich-graue Geschwulstmasse setzt sich aus verschieden dicken, sich in allen möglichen Richtungen durchflechtenden grauweissen Bündeln zusammen, zwischen welchen ein filzartiges Gewebe sich befindet. Makroskopisch erinnert das Bild sehr an ein leicht ödematöses Fibrom. Die centralen und peripheren Partien verhalten sich in ihrem Aussehen völlig gleich.

Im unteren Pole der Geschwulst findet sich ein etwa haselnussgrosser, 20:12 mm messender. Herd von weicher bräunlicher Beschaffenheit. Von dem umliegenden Gewebe ist dieser Herd durch eine etwa $1 \mathrm{~mm}$ dicke fibröse Kapsel abgesetzt." Ausserdem finden sich, in dem Innern des. Tumors zerstreut, mehrere erbsengrosse Cystchen mit klarem Inhalt, deren Wand von einem dicken, weissgrauen, theilweise verkalkten Gewebe gebildet wird.

Die mikroskopische. Untersuchung ergab an den von den verschiedensten Stellen stammenden Schnitten im Wesentlichen ein übereinstimmendes Bild. In den peripheren Partien lässt sich eine Kapsel aus kernarmem Bindegewebe. erkennen, von welcher aus hier und da Züge ins Innere der Geschwulst ausstrahlen. Die Hauptmasse der Geschwulst bilden eigenthümliche Fasern, welche zum Theil zu verschieden dicken Bündeln angeordnet sind, zum Theil auch mit spärlichem fibrillären Bindegewebe untermischt, regellos sich verflechten und so einen dichten Filz bilden. Zwischen den Faserbündeln findet sich vielfach ein lockeres, etwas ödematöses, wenig kernreiches Bindegewebe in mässiger Menge, an den 
-meisten Stellen der Geschwulst ist das Bindegewebe nur in ganz geringer Menge vorhanden und tritt an Masse gegen die erwähnten Fasern sehr zurück.

Die zu Bündeln vereinigten Fasern zeigen an Längsschnitten im Allgemeinen einen streng parallelen Verlauf, die Fasern sind vielfach gewellt, durchflechten und überkreuzen sich aber im Allgemeinen innerhalb der Bündel nicht.

Die einzelnen, die Bündel zusammensetzenden Fasern sind sehr dünn, im Allgemeinen von gleich bleibender Breite, hier und da leichte Anschwellungen aufweisend. Häufig lässt sich eine Faser in einem und demselben Schnitte auf eine ziemlich grosse Strecke verfolgen und kann man dann feststellen, dass ein Auslaufen der Faser in eine Spitze niemals statt hat. Zwischen den einzelnen Fasern sind dann und wann feine Bindegewebsfibrillen wahrzunehmen. Den Fasern angelagert sind zahlreiche längliche, stäbchenförmige Kerne, welche an die glatter Muskelfasern erinnern.

Die Querschnitte stärkerer Bündel bilden polygonale Felder, welche bei mittlerer Vergrössering wie punktirt aussehen mit einzelnen unregelmässig eingestreuten Querdurchschnitten länglicher Kerne. Die einzelnen Punkte entsprechen den einzelnen das Bündel bildenden Fasern und lässt sich an diesen Stellen leicht darthun, dass die Kerne nicht im Innern der Fasern, sondern an deren Peripherie gelagert sind.

Bindegewebige Scheidewände finden sich im Innern stärkerer Bündel nur selten und in grösseren Abständen; die feineren Bündel lassen solche vollständig vermissen. Dagegen ist eine dünne Lage lockeren Bindegewebes öfters an der Aussenfläche der Bündel und zwischen den einzelnen Fascikeln zu sehen. Von den stärkeren Bündeln zweigen öfters feinere ab, welche ihrerseits wieder mit benachbarten Bündeln zusammenhängen, so dass stellenweise ein plexiformes Bild zu Stande kommt.

Die soeben beschriebenen Faserbündel machen durchaus den Eindruck markloser Nervenfasern; von glatter Muskulatur sind sie durch ihre bedeutende Länge, das Verhalten der Kerne, die Verästelung und namentlich auch das Färbeverhalten unterschieden. Nach der van Gieson'schen Färbung zeigen die Fasern einen schwach gelblichen, hier und da auch leicht röthlichen Ton, welcher mit dem Rubinroth des collagenen Bindegewebes und dem Strohgelb der glatten Muskulatur gar nicht zu verwechseln ist. Das 
mächtige quantitative Ueberwiegen der marklosen Nervenfasern über das Bindegewebe tritt namentlich bei dieser Färbung sehr prägnant hervor.

Eine beinahe noch schärfere Trennung der Remak'schen Fasern von dem Bindegewebe ermöglicht die Mallory'sche Färbung. Die marklosen Nervenfasern nehmen bei dieser Färbung einen rein blauen Ton an, während sich das Bindegewebe viel intensiver und schmutzig-violett färbt.

Schon bei den gewöhnlichen Färbemethoden begegnet man allenthalben markhaltigen Nervenfasern in geringer Zahl; sie sind an den einzelnen Stellen der Geschwulst in sehr verschiedener Reichlichkeit vorhanden, fehlen aber nirgends vollständig. Sie sind mit den markhaltigen Fasern des, Rückenmarks und der peripheren Nerven verglichen, auffallend dünn, zeigen ganz gewöhnlich vielfach unterbrochene Markscheiden, welche die Weigert'sche Färbung sehr prompt annehmen.

Ausserdem finden sich von nervösen Elementen sehr zahlreiche Ganglienzellen in allen Theilen der Geschwulst. Dieselben sind an den einzelnen Punkten gleichfalls in sehr verschiedener Reichlichkeit anzutreffen, doch ist auch an den Stellen, wo sie nur relativ spärlich vertreten sind, kein Gesichtsfeld, in welchem nicht die eine oder andere Ganglienzelle gelegen wäre.

Periphere und centrale Zonen der Geschwulst zeigen auch hinsichtlich der Vertheilung und Häufigkeit der Ganglienzellen keine Unterschiede. Die Ganglienzellen sind zum Theil haufenweise zusammengelagert, wie man dies in den spinalen und sympathischen Ganglien findet; oder sie sind mehr vereinzelt regellos in dem Gewirre markloser Fasern verstreut, oder endlich finden sie sich als Einlagerungen in stärkeren Bündeln Remak'scher Fasern. An den Stellen, wo die Ganglienzellen in Haufen beisammen liegen, pflegen auch die markhaltigen Fasern in grösserer Zahl vorhanden zu sein. Diese Zellen stellen kuglige oder elliptische, manchmal auch etwas abgeplattete Körper von sehr verschiedener Grösse dar, deren Durchmesser von 28-125 $\mu$ schwanken. Sie sind, durchweg von einer feinen Hülle umschlossen; mit zablreichen, endothelartigen Kernen, welche auch auf die von den Zellen entspringenden Fortsätze übergehen. Derartige Fortsätze sind an ziemlich zahlreichen Zellen aufzufinden. Das Protoplasma ist feinkörnig, selten mehr homogen; es nimmt die Kernfärbemittel meist nur schwach an. 
Pigmentablagerung habe ich in dem Protoplasma nirgends beobachten können; öfters ist in demselben Vacuolenbildung zu erkennen. Der grosse, meist excentrisch gelagerte Kern nimmt die Farbstoffe nur wenig stärker aut als das Protoplasma, dagegen färbt sich das grosse Kernkörperchen sehr interisiv.

Ziemlich häufig enthalten die Zellen 2 Kerne, eine grössere Anzahl Kerne habe ich in einer Zelle jedoch nie auffinden können. An manchen Stellen, wo die Zellen sehr dicht gelagert sind, hat man den Eindruck, als ob mehrere Zellen (3-4, es betrifft dies ausnahmslos kleinere Exemplare) in einer gemeinsamen Scheide gelegen seien. Sonstige direct auf eine Proliferation der Ganglienzellen hinweisende Anzeichen, insbesondere Kerntheilungsfiguren, sind nicht zu erheben. Auffallend ist vielfach die Intensität, mit welcher das Protoplasma der Ganglienzellen den Farbstoff der Weigert'schen Markscheidenfärbung, auch bei sehr langer Differenzirung mit Borax-ferricyankalilösung zurückhält; dagegen konnte jeh nirgends einen deutlichen Markmantel an den von den Ganglienzellen entspringenden kortsätzen nachweisen.

Die Vascularisation der Geschwulst ist eine mässig reichliche; die stärkeren Gefässstämme zeigen ausgesprochene endarteritische Veränderungen; an der Aussenseite kleiner Gefässe sind häufig kleine Rundzellinfiltrate zu sehen, welche auch sonst hier und da in der Geschwulst, entfernt von den Gefässen, anzutreffen sind.

Mehrfach sind kleine Kalkablagerungen im Tumorgewebe vorhanden; einzelne Kalkkörner sind von einer kernhaltigen Hülle umgeben, welche derjenigen der Ganglienzellen gleicht. Dies legt den Gedanken nahe, dass die Kalkablagerungen, z. Th. wenigstens, Ganglienzellen entsprechen.

Der erwähnte haselnussgrosse Herd im unteren Pole des Tumors besteht aus einer peripheren Zone eines sehr dichten, kernarmen Bindegewebes, welches Hämatoidinkörner, sowie Kalkablagerungen einschliesst.

Der Inhalt besteht aus in Organisation begriffenen hyalinen Fibrin, erhaltenen rothen Blutkörperchen und Resten von solchen, sowie einer feinkörnigen Gerinnungmasse.

Die erwähnten Cystchen stellen im Innern erweichte Fibrome dar. Die poripheren Wandzonen bestehen aus dichtem, kernarmen, häufig verkalktem fibrillärem Bindegewebe, während im Centrum zarte Fasern mit grossen sternförmigen, häufig mit körnigem gelbem Pigment beladene, stark gequollene Zellen sich finden. Zwischen 
den Fasern und Zellen ist eine feinkörnige, mit Hämatoxylin stark blau sich färbende Gerinnungsmasse abgelagert.

Die Deutung der Geschwulst kann nach der vorstehenden Beschreibung nicht zweifelhaft sein: es handelt sich um ein gangliöses Neurom mit vorwiegend marklosen Nervenfasern und zwar um ein Neuroma verum, bei dessen Aufbau der bindegewebige Antheil der Geschwulst vollkommen in den Hintergrund tritt:

Herr Professor Beneke in Brannschweig und Herr Stadtarzt Dr. Knauss in Stuttgart hatten die grosse Güte, mir Präparate der von ihnen beschriebenen Neurome zu überlassen und konnte ich an diesen Präparaten die weitgehende Uebereinstimmung im Baue mit der hier geschilderten Geschwulst feststellen.

Den Ausgangspunkt des Tumors bildet der Sympathicus; hierfür spricht der ganze Bau der Geschwulst, das starke Ueberwiegen der marklosen Nervenfasern and die eigenthümliche Beschaffenheit der" markhaltigen Fasern, welche den normalerweise in den sympathischen Ganglien und dem Grenzstrange anzutreffenden vollkommen entsprechen. Gegen den Ausgang von den spinalen Nervenwurzeln spricht einmal die Lage des Tumors und das vollkommene Fehlen eines Stieles mit stärkeren Nervenstämmen.

Welcher Theil des Sympathicus den Ausgangspunkt bildete, lässt sich mit Bestimmtheit nicht angeben, da der topographisch genaue Sitz der Geschwulst bei der Operation aus leicht begreiflichen Gründen nicht zu bestimmen war. Die Geschwulst entsprach dem Sitze der Plexus mesenterici; vielleicht lieferte ein die mehrfach erwähnte Arterie umspinnender Theil eines Plexus mesentericus das Bildungsmaterial für das Neoplasma.

Bei der grossen Menge der Ganglienzellen und der marklosen Nervenfasern muss wohl angenommen werden, dass beide Elemente, zum. grossen Theile wenigstens, neugebildet sind, wennschon der directe Nachweis der Proliferation auch in unserem Falle nicht zu erbringen ist. Die annähernd gleichmässige Vertheilung der Ganglienzellen in allen Theilen der Geschwulst zusammen mit ihrer grossen Zahl und dem Umstande, dass sie sich auch im Innern der Nervenbündel finden, spricht dagegen, dass es sich um ein einfaches Auswachsen präexistenter Nervenfasern nach Analogie der sog. Amputationsneurome handelt und dass die Ganglienzellen, ohne selbst neugebildet zu sein, lediglich aus präexistenten Gruppen solcher durch einen Auffaserungsprocess in der geschilderten Weise in der ganzen Geschwulst sich vertheilt hätten. 
208 Glockner, Ueber einen Fall von Neuroma verum gangliosum ete.

Auch der Umstand, dass die Ganglienzellen durchweg frei von Pigment befunden wurden, deutet nach den Untersuchungen Graupner's ${ }^{1}$ ) daraut hin, dass es sieh um jugendliche Zellindividuen handelte.

Klinisch interessant waren die schweren Erscheinungen, welche die Patientin in der auf die Operation folgenden Nacht darbot und welche an das Bild einer schweren inneren Blutung resp. an acute Darmlähmung erinnerten. $O b$ und wieweit hierfür ein bei der Operation gesetztes Trauma des Sympathicus anzuschuldigen ist, entzieht sich allerdings bei dem vollständigen Mangel an Kenntnissen über die physiologische Bedeutung des Bauchsympathicus unserer Beurtheilung.

\section{Erklärung der Abbildungen auf Tafel.VII.}

Figur 1. An Ganglienzellen reiche Partie aus den centralen Theilen der Geschwulst mit zahlreichen markhaltigen Nervenfasern. Markscheidenfärbung nach Weigert. Leitz, Obj. 4, Ocul. 1.

Figur 1a. Einige markhaltige Fasern bei stärkerer Vergrösserung. Man sieht dentlich die häufigen Lücken im Markmantel. Markscheidenfärbung. Zeiss DD, Obj. 2.

Figur 2. Ganglienzellen bei stärkerer Vergrösserung. Deutliche Fortsätze an einzelnen Zellen. Hämatoxylin-Eosin. Zeiss DD, Obj. 3. Ocul. 2.

Figur 3. Vorwiegend längs getroffene Bündel markloser Nervenfasem. Dazwischen lockeres Bindegewebe. van Gies on'sche Färbung. Leitz, Obj. 4, Ocul. 1 .

Figur 4. Marklose Nervenfasern bei stärkerer Vergrösserung. Eine Ganglienzelle in einem Bündel eingelagert. Mall ory' sche Färbung. Zeiss DD, Ocul. 2.

1) Beiträge zur normalen and pathol. Anatomie des sympath. Nervensystems. Ziegler's Beiträge. Bd. XXIV. 

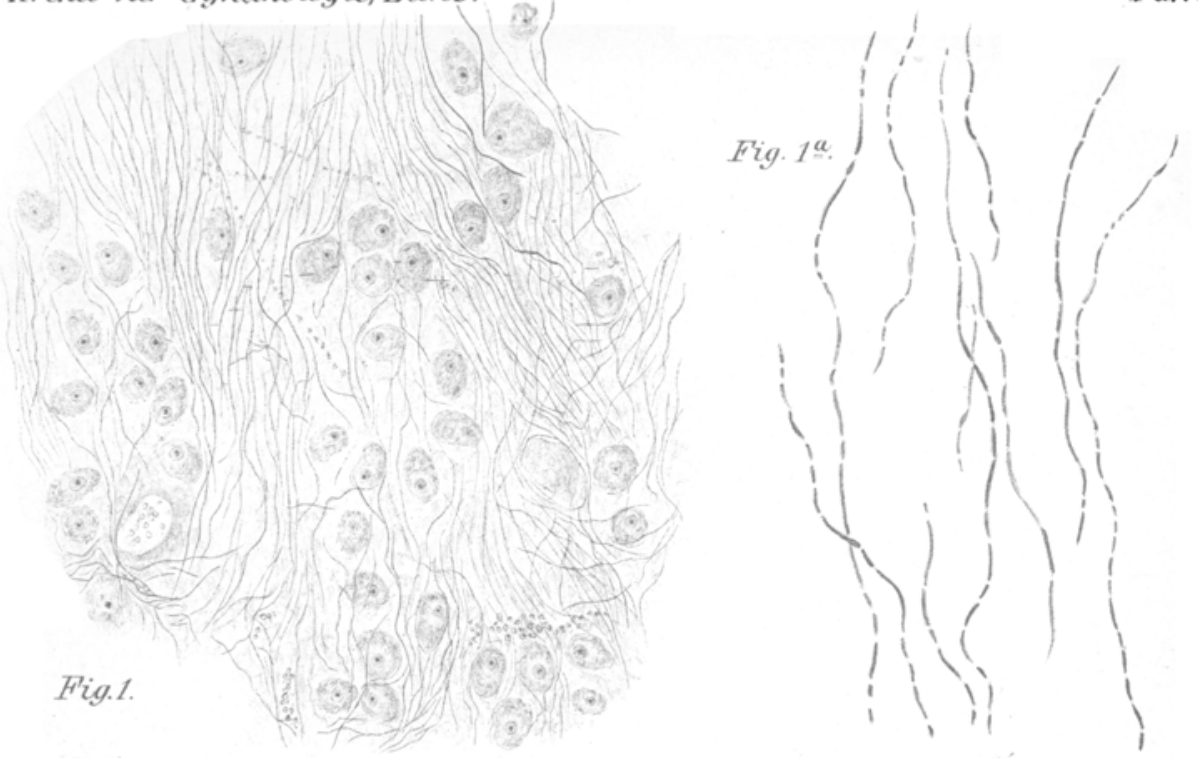

Fig. 2.

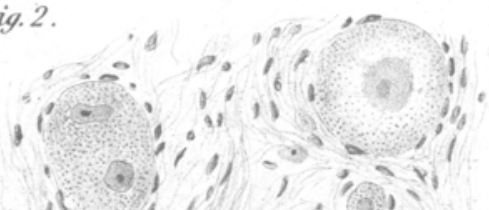

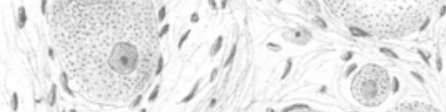

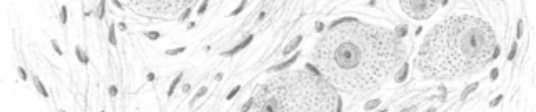

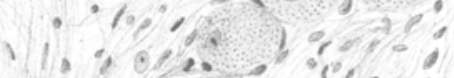

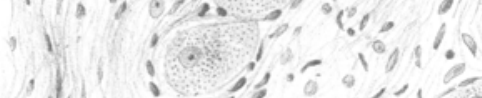

i) $1:=0 ?:-1,3$

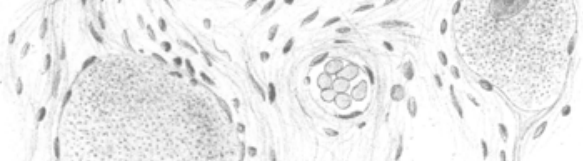

Fig.3.

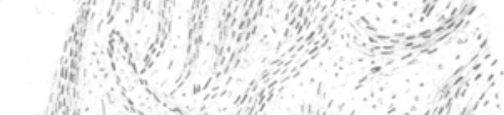

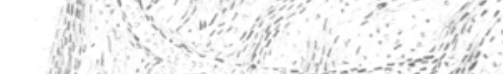

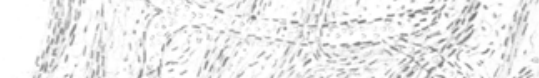

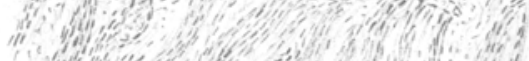

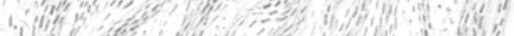
保

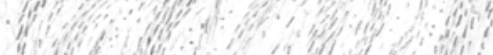

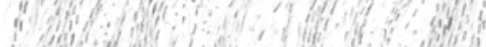
(n) its 